\title{
Material Deposition by a Soft-Landing of Mixed Ar Gas Cluster Projectiles at the Ag (111) Surface
}

\author{
D. MaCiażéE* And Z. Postawa
}

Institute of Physics, Jagiellonian University, S. Łojasiewicza 11, 30-348 Kraków, Poland

\begin{abstract}
Molecular dynamics computer simulations are employed to investigate the possibility to deposit organic molecules of octane and $\beta$-carotene by a soft-landing of $\mathrm{Ar}$ mixed gas cluster projectiles at the $\mathrm{Ag}(111)$ surface at an incident angle of $45^{\circ}$. The effect of a projectile composition and its kinetic energy on the efficiency of molecular deposition is investigated. It is shown that deposition of organic material from mixed gas clusters is possible for kinetic energy below $8 \mathrm{keV}$, which corresponds to approximately $0.02 \mathrm{eV} /$ nucleon in a cluster consisting of 10000 argon atoms.
\end{abstract}

DOI: 10.12693 /APhysPolA.136.260

PACS/topics: 07.05.Tp, 31.15.xv, 81.15.-Z

\section{Introduction}

Soft and reactive landing of mass-selected clusters onto surfaces have become a topic of substantial interest due to their promising potentials for the highly controlled preparation of materials. These deposition techniques overcome many of the limitations associated with conventional thin-film production techniques and offer unprecedented selectivity and specificity of preparation of deposited species [1,2]. For instance, soft-landing has been used extensively for deposition of nanoclusters and biological molecules. The structure and properties of the resulting materials are controlled by the properties of the projectile and the chemical properties of the surface. The critical parameter of soft-landing is the kinetic energy of deposited clusters. This energy should be kept at a low value to prevent substrate and nanocluster reorganization [1]. It has been observed that the kinetic energy range for soft-landing can be significantly extended by application of weakly bound overlayers, which allow the deposited cluster to dissipate its kinetic energy gently. It is accomplished, for instance, by depositing a thin buffer layer composed of rare-gas atoms at the surface $[1,3]$. In principle, the same action could be achieved by embedding deposited entities inside the noble gas cluster projectiles. Application of such concept would also allow increasing the primary kinetic energy, as most of this energy would be carried away by nonbonding rare-gas atoms. Higher primary kinetic energy would allow working with more focused beams, eliminating a drawback of standard soft-landing approaches associated with a lack of spatial control over deposited species $[1,2]$.

\footnotetext{
* corresponding author; e-mail: dawid.maciazek@doctoral.uj.edu.pl
}

In this paper, we test this concept by investigating the impact of mixed octane/Ar and $\beta$-carotene/Ar cluster projectiles on the $\operatorname{Ag}(111)$ substrate. The effect of the primary kinetic energy, the molecular size, and the cluster composition on the deposition efficiency of organic molecules and on the molecular fragmentation is investigated.

\section{Computer model}

A detailed description of the molecular dynamics computer simulation used to model cluster bombardment can be found elsewhere [4]. Briefly, the motion of the particles is determined by integrating Hamilton's equations of motion. The forces among the particles are described by a blend of pairwise additive and many-body potential energy functions. The interactions among $\mathrm{C}$ and $\mathrm{H}$ atoms are described by adaptive interatomic reactive bond order potential (AIREBO) [5]. Embedded atom potential (EAM) is used to describe the $\mathrm{Ag}-\mathrm{Ag}$ interactions [6]. The $\mathrm{Ar}-\mathrm{Ar}$ [7], $\mathrm{Ar}-(\mathrm{C}, \mathrm{H})$, and $\mathrm{Ag}-(\mathrm{Ar}, \mathrm{C}, \mathrm{H})$ [8] interactions are described by a pairwise Lennard-Jones potential with appropriate parametrizations. These interactions result in average binding energy of $0.07 \mathrm{eV}$ for an $\mathrm{Ar}$ atom, and $0.8 \mathrm{eV}$ and $4.4 \mathrm{eV}$ for octane and $\beta$-carotene molecules, respectively.

The shape and size of the sample were chosen based on visual observation of the cluster impact. As a result, a hemispherical shape with a radius of $15 \mathrm{~nm}$ is chosen to absorb impact wave propagation best. In order to capture the sliding motion of molecules from atomized cluster additional cuboid volume was added to the hemisphere with dimensions of $200 \mathrm{~nm}$ $\times 150 \mathrm{~nm} \times 3 \mathrm{~nm}$. Rigid and stochastic regions with a thickness of 0.3 and $1.7 \mathrm{~nm}$, respectively, were used around the sample to preserve the shape of the system and to simulate thermal bath that keeps the sample at $T=0 \mathrm{~K}$ and helps inhibit the pressure wave reflection from the system boundaries [9]. Argon gas 
clusters containing organic molecules with mass concentration of $2 \%, 4 \%, 8 \%$, and $12 \%$ were created with Packmol package [10] and then thermalized at $T=50 \mathrm{~K}$ for $1 \mathrm{~ns}$ using the Langevin thermostat [11]. The projectile impact angle was 45 degrees, and its kinetic energy was varied between 2 and $35 \mathrm{keV}$. Octane $\left(\mathrm{C}_{8} \mathrm{H}_{18}\right)$ and $\beta$-carotene $\left(\mathrm{C}_{40} \mathrm{H}_{56}\right)$ molecules were chosen to probe the effect of the molecular size on the deposition efficiency. All projectiles had the same mass corresponding to the mass of Ar cluster consisting of 10000 Ar atoms. In other words, they had the same velocity at a given kinetic energy. The simulations were run in an NVE ensemble and extended up to $80 \mathrm{ps}$, which was long enough to achieve saturation in the coverage. Simulations were performed with the large-scale atomic/molecular massively parallel simulator (LAMMPS) code [12]. Nine randomly selected impact points located near the center of the sample were chosen to achieve statistically reliable data.

\section{Results and discussion}

The fractions of deposited organic molecules for octane and $\beta$-carotene doped argon gas clusters with a different mass composition as a function of the cluster initial kinetic energy are shown in Fig. 1. The general shape of this curve is similar for octane and $\beta$-carotene regardless of their different sizes. Such behavior can be

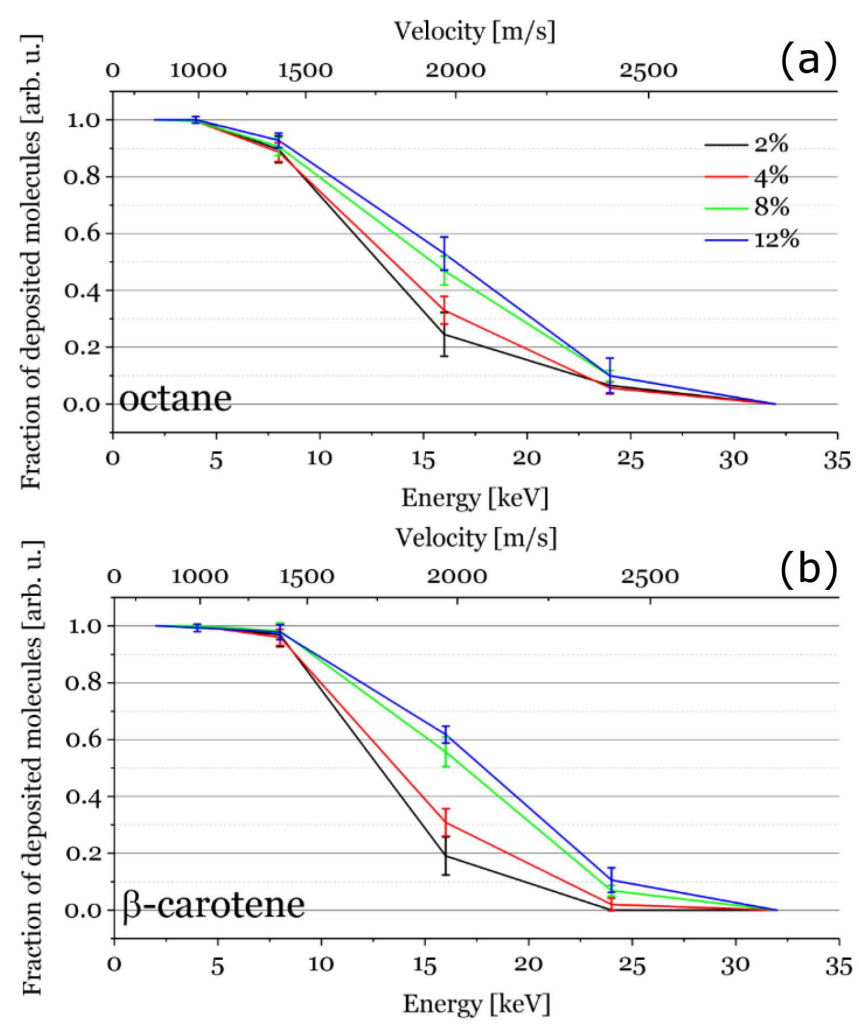

Fig. 1. Dependence of a fraction of deposited molecules on the initial kinetic energy for argon clusters with different composition of (a) octane and (b) $\beta$-carotene. attributed to a similar scaling of the primary kinetic energy of the molecules inside the projectiles, and their binding energy to the surface with the size of the molecule. At low projectile kinetic energy almost all organic molecules are adsorbed at $\mathrm{Ag}(111)$. The deposition probability begins to decrease at higher kinetic energy and drops to nearly zero at $35 \mathrm{keV}$. At kinetic energy below $8 \mathrm{keV}$, which corresponds to $0.02 \mathrm{eV} /$ nucleon, the fraction of deposited molecules does not depend on the molecular concentration. This parameter begins to influence the fraction of adsorbed molecules above approximately $8 \mathrm{keV}$. Projectiles having a larger number of organic molecules deposit a larger fraction of molecules. In all investigated cases, no ejection of substrate atoms is observed.

Temporal evolution of projectile impact containing $12 \%$ of $\beta$-carotene is shown in Fig. 2 for 8 and $24 \mathrm{keV}$ projectiles. In both cases, the impinging projectile deforms immediately after the impact adopting a flat ellipsoidal shape. The molecules move slowly near the surface during $8 \mathrm{keV}$ impact. The interaction is long, and the molecules have enough time to slow down. Almost all molecules remain at the surface. The increase in the primary kinetic energy has a twofold effect on the behavior of impinging molecules. Firstly, the kinetic energy stored in their movement is increased. Consequently, the interaction time of molecules with the surface is reduced and they have less time to lose kinetic energy. As a result, a smaller fraction of these molecules is deposited. In addition, collisions between the molecules and the Ag surface become violent, which leads to a molecular fragmentation and a further decrease in the number of deposited molecules.

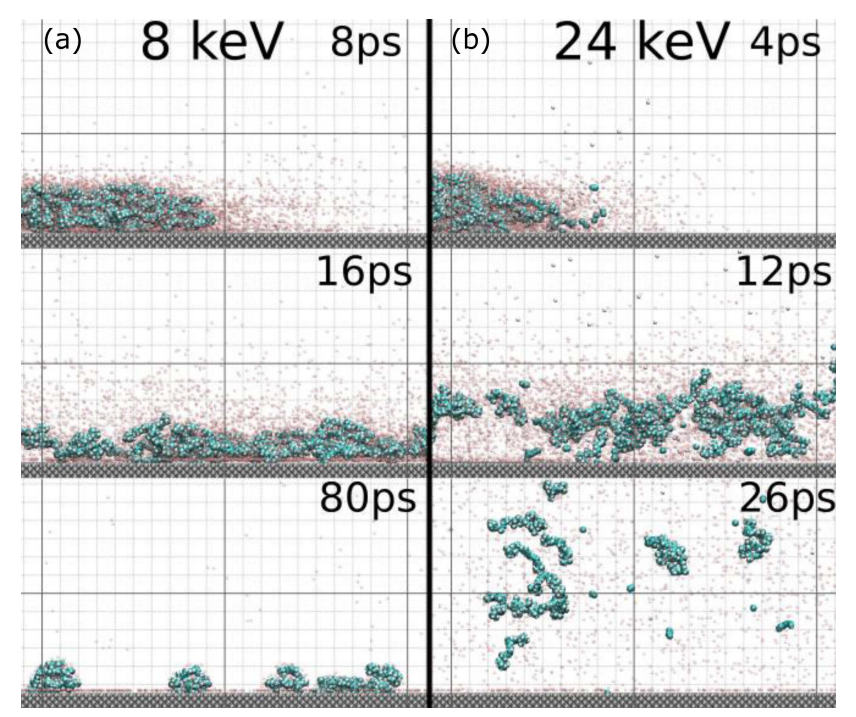

Fig. 2. Cross-sectional view of temporal evolution of deposition event for $12 \% \operatorname{Ar} / \beta$-carotene projectile with kinetic energy of (a) $8 \mathrm{keV}$ and (b) $24 \mathrm{keV}$. Ar atoms and atoms forming a molecule are represented as small and large spheres, respectively. Continuous and dotted grid lines are separated by $10 \mathrm{~nm}$ and $1 \mathrm{~nm}$, respectively. 
Temporal evolution of the $2 \%$ and $12 \% \operatorname{Ar} / \beta$-carotene projectiles impinging $\mathrm{Ag}$ (111) surface with a kinetic energy of $16 \mathrm{keV}$ is shown in Fig. 3 to investigate a strong dependence of the deposited molecular fraction on the projectile concentration observed above $8 \mathrm{keV}$. At this kinetic energy, the interaction time is reduced. For a $2 \%$ projectile, only a few molecules remain close to the surface long enough to become decelerated by an interaction with the surface. In the case of a $12 \%$ projectile, a larger number of molecules get in contact with the $\mathrm{Ag}(111)$ surface. Furthermore, these molecules interact also with other molecules that do not have contact with the surface. Due to these indirect interactions, some of these molecules can also be slowed down and remain attached to the surface. The process leads to a formation of clustered molecular nanostructures deposited at the surface.

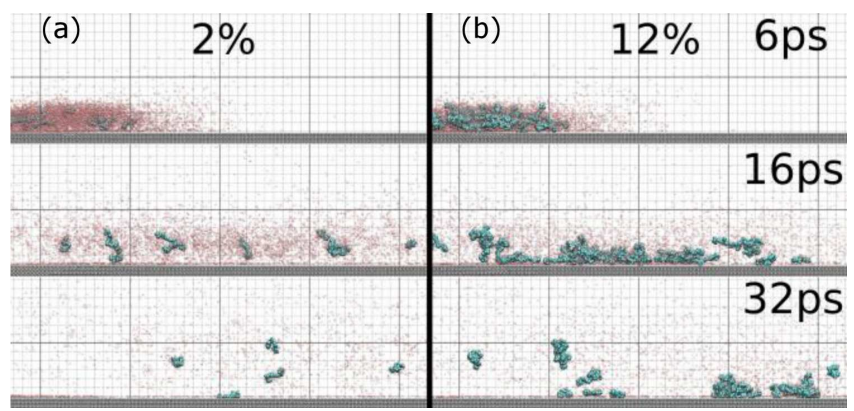

Fig. 3. Cross-sectional view of temporal evolution of the deposition event for (a) $2 \%$ and (b) $12 \%$ $\mathrm{Ar} / \beta$-carotene projectile impacts with $16 \mathrm{keV}$ kinetic energy. Ar atoms and atoms forming a molecule are represented as small and large spheres, respectively. Continuous and dotted grid lines are separated by $10 \mathrm{~nm}$ and $1 \mathrm{~nm}$, respectively.

\section{Conclusions}

The possibility of deposition of organic molecules at the $\operatorname{Ag}(111)$ surface has been investigated using molecular dynamics computer simulations. It has been shown that organic molecules can be deposited on the surface by this approach. The size of the molecule has a small effect on the fraction of deposited molecules. However, this quantity strongly depends on the initial kinetic energy of the projectile. Such behavior is attributed to a decrease of the interaction time of molecules with the surface and to the increase of the primary kinetic energy above the surface bounding energy limit. Above certain kinetic energy, the fraction of deposited molecules also depends on the projectile composition. Effect of indirect interaction through intermolecular forces is responsible for such behavior.

\section{Acknowledgments}

The authors gratefully acknowledge financial support from the Polish National Science Center, Program no. 2015/19/B/ST4/01892. This research was supported in part by PL-Grid Infrastructure.

\section{References}

[1] V.N. Popok, I. Barke, E.E.B. Campbell, K.H. MeiwesBroer, Surf. Sci. Rep. 66, 347 (2011).

[2] P.M. Dinh, P.G. Reinhard, E. Suraud, Phys. Rep. 485, 43 (2010).

[3] H.P. Cheng, U. Landman, Science 260, 1304 (1993).

[4] B.J. Garrison, Z. Postawa, in: ToF-SIMS-Surface Analysis by Mass Spectrometry, 2nd ed., Eds. J.C. Vickerman, D. Briggs, IMP \& Surface Spectra Ltd, Chichester 2013, p. 151.

[5] S.J. Stuart, A.B. Tutein, J.A. Harrison, J. Chem. Phys. 112, 6472 (2000).

[6] P.L. Williams, Y. Mishin, J.C. Hamilton, Model. Simul. Mater. Sci. Eng. 14, 817 (2006).

[7] J.A. White, J. Chem. Phys. 111, 9352 (1999).

[8] R. Chatterjee, Z. Postawa, N. Winograd, B.J. Garrison, J. Phys. Chem. B 103, 151 (1999).

[9] Z. Postawa, B. Czerwinski, M. Szewczyk, E.J. Smiley, N. Winograd, B.J. Garrison, Anal. Chem. 75, 4402 (2003).

[10] L. Martínez, R. Andrade, E.G. Birgin, J.M. Martinez, Comput. Chem. 30, 2157 (2009).

[11] T. Schneider, E. Stoll, Phys. Rev. B 17, 1302 (1978).

[12] S. Plimpton, J. Comput. Phys. 117, 1 (1995). 